\title{
Filigrane
}

Écoutes psychothérapiques

\section{La chair à vif}

\section{Rose Dymetryszyn}

Volume 18, numéro 2, automne 2009

Le corps. Sur le divan. Dans le fauteuil II

URI : https://id.erudit.org/iderudit/039287ar

DOI : https://doi.org/10.7202/039287ar

Aller au sommaire du numéro

Éditeur(s)

Revue Santé mentale au Québec

ISSN

1192-1412 (imprimé)

1911-4656 (numérique)

Découvrir la revue

\section{Citer cet article}

Dymetryszyn, R. (2009). La chair à vif. Filigrane, 18(2), 31-37.

https://doi.org/10.7202/039287ar

\section{Résumé de l'article}

Par le truchement d'une présentation clinique, l'auteur tente de situer la place du corps en psychanalyse. Divers auteurs psychanalytiques sont invoqués de la relation mère-enfant de la toute petite enfance jusqu'aux séquelles post-traumatiques lorsque le corps est ressenti comme objet fragmenté, objet partiel persécuteur, source de sensations diffuses inexplicables ou enjeu de conflits psychiques.
Ce document est protégé par la loi sur le droit d'auteur. L'utilisation des services d’Érudit (y compris la reproduction) est assujettie à sa politique d'utilisation que vous pouvez consulter en ligne.

https://apropos.erudit.org/fr/usagers/politique-dutilisation/ 


\title{
La chair à vif
}

\author{
rose dymetryszyn
}

Par le truchement d'une présentation clinique, l'auteur tente de situer la place du corps en psychanalyse. Divers auteurs psychanalytiques sont invoqués — de la relation mère-enfant de la toute petite enfance jusqu'aux séquelles post-traumatiques lorsque le corps est ressenti comme objet fragmenté, objet partiel persécuteur, source de sensations diffuses inexplicables ou enjeu de conflits psychiques.

\begin{abstract}
By way of a case presentation, the author attempts to discuss the place of the body in psychoanalysis. Several psychoanalytic authors are invoked from theorists of the earliest mother-infant relationship to the post-traumatic consequences when the body is then felt as a fragmented object, a partial persecutory object, a source of confused, unexplainable sensations or the playing field of psychic conflicts.
\end{abstract}

En considérant la place du corps en psychanalyse, je suis particulièrement saisie par le dernier paragraphe d'un article de Gantheret:

«Dans la clinique comme dans la théorie, nous sommes donc amené à considérer que le champ psychanalytique s'établit dans une coupure, qui laisse à l'un de ses bords le corps biologique, pour en assurer "de l'autre côté" la reprise dans un langage, dans un système de signes. Cette coupure, c'est aussi celle qui sépare le psychotique de l'accès au symbole...» (Gantheret, 1971,146)

Ma première rencontre avec Kronos se fait en milieu intermédiaire, la salle d'urgence. Kronos, un jeune homme de 17 ans vient tout juste d'être recousu par un généraliste. Dans un état dissocié, il s'est entaillé l'avant-bras avec un couteau exacto pendant qu'il dessinait sur du carton. Dans le passé, toutes les coupures ont été profondes et ont nécessité plusieurs sutures de la peau et des tissus sousjacents. Kronos dit n'avoir aucun souvenir d'avant la césure et ne peut pas articuler clairement ce qui a précipité son épisode d'automutilation. Son accompagnateur, un superviseur en milieu résidentiel thérapeutique pour adolescents, l'a aperçu et a fait venir l'ambulance. Cet homme qui l'accompagne se demande si la séparation qui s'annonce n'est pas l'événement stressant précipitant l'entaille. J'apprends que bientôt, lors de ses dix-huit ans, Kronos devra emménager ailleurs.

De son histoire, l'accompagnateur souligne qu'il a été admis en milieu thérapeutique suite à l'intervention de l'aide à l'enfance, il y a trois ans. Auparavant, Kronos vivait dans les rues de la grande ville.

J'offre de le rencontrer régulièrement avec le but manifeste de l'aider à se séparer de sa maison thérapeutique et de lui donner un soutien régulier indépendant du lieu où il décidera d'habiter. Au moment de mon offre, je ne suis pas sûre 
de ce que Kronos pourra en faire puisqu'il ne semble pas avoir une bonne notion du temps, il se retrouve à l'urgence régulièrement et les nouvelles relations sont testées souvent avant que ne s'établisse une stabilité permettant le travail de psychothérapie. Par après, je ne suis plus sûre de ce que je pourrai en faire, réalisant que je suis en début de carrière, dans un statut «d'apprenti-sorcier en quête de sens» (Aulagnier, 2001, 59), c'est-à-dire en apprentissage de la psychothérapie psychodynamique. Néanmoins, je me sens dans l'obligation de relever le défi posé par ce jeune homme en très grande souffrance sans mot.

Malgré les couches successives d'incidents traumatiques, Kronos révèle: un potentiel intellectuel considérable - malgré les lacunes sérieuses de son éducation, il termine en un an l'équivalent de son secondaire avec l'aide d'un tuteur et des leçons à l'ordinateur; une bonne capacité à observer les gens et un grand désir à vouloir exprimer et comprendre ce qui se passe en lui ; un humour noir qui l'aide des fois à initier des échanges verbaux et paradoxalement à se protéger par son mordant; des talents artistiques considérables où il peut exprimer plus aisément son vécu dans le dessin et dans la chanson.

Un an plus tard, mon offre de soutien deviendra psychothérapie psychanalytique à raison de trois rencontres régulières par semaine, et durera à peu près cinq ans.

Gantheret note :

«Une triple caractéristique attachée à la question du corps en psychanalyse: marginale et frontalière d'une part [les lettres de Freud à Fliess, L'Esquisse], fondatrice et constitutive d'autre part [Études sur l'hystérie, Le Moi et le Ça; les écrits de Federn, Schilder et Reich], enfouie, recouverte enfin. C'est assez pour nous laisser entendre que quelque chose de l'ordre de la naissance, de la question des origines, est ici en jeu. (Gantheret, 1971, 138)

La première césure, la naissance, sépare radicalement le nourrisson du corps de sa mère. Une série d'auteurs - Aulagnier, Bick, Bion, Fonagy, Stern, Tustin, Winnicott — tentent d'élaborer à partir du couple mère-nourrisson les ingrédients et les processus nécessaires pour développer une capacité à être seul pour penser et une capacité à symboliser. D'abord en détresse, dans une succession d'états instables somatiques sensoriels, le nourrisson est calmé et satisfait dans ses besoins par la mère par l'intermédiaire d'un état maternel que Bion appelle «rêverie», qui relève à la fois du somatique et du cognitif. L'état de détresse du bébé induit chez sa mère, par une forme primitive d'identification projective, un état de réflexion lui permettant de localiser la source de détresse, de transformer cette détresse en quelque chose de moins désorganisé et désorganisateur et ensuite de communiquer cette transformation structurante et calmante par son toucher, sa voix, son regard et par ses soins administrés au corps du nourrisson. Le processus 
de structurer ce qui est chaotique est la définition que donne Bion à la «contenance », un concept qui désigne la mère - celle qui exerce la fonction de contenir, le contenant — et le nourrisson, celui qui est contenu. Lorsque le bébé éprouve plusieurs expériences d'être contenu suffisamment bien, il développe sa propre capacité de transformer le chaos interne en un éprouvé contenu, ultimement la capacité de penser et de symboliser le corps.

Par contre, si la mère est troublée suffisamment par ce que son bébé lui transmet de façon non verbale, qu'elle ne peut contenir et transformer son état désorganisé et qu'elle lui offre plutôt son propre vécu intolérable, le bébé est menacé dans l'intégration de soi et reste pris dans le concret, très près de l'expérience physique.

L'échec d'expériences précoces suffisamment bonnes force l'enfant mal équipé à éprouver une terreur sans nom, la catastrophe psychique et les angoisses primitives sans espoir d'être soulagé. L'enfant s'en défendra en concrétisant son expérience: couper la peau pour représenter la faiblesse éprouvée envers cette limite du dedans et du dehors, du soi et du non-soi; recourir au processus de dépersonnalisation, de déréalisation, de dissociation; l'incapacité à être seul pour réfléchir ; l'identification adhésive pathologique (Meltzer in Lebel, 2006 , 21) avec sa recherche effrénée d'un objet avec lequel se fusionner et avec des préoccupations compulsives intenses; la recherche d'objets transitionnels durs pour palier à sa peau-passoire et pour colmater tant bien que mal les angoisses de ne plus exister, de se vider, de se liquéfier; la recherche de sensations fortes suivant des comportements à risque, les conduites toxicomaniaques et le surinvestissement de l'endurance physique pour se sentir vivant.

De par son apparence, Kronos intimide les gens. Il est grand, très mince, presque tout le temps vêtu d'une veste noire en cuir épais où sont fixées plusieurs chaînes métalliques lourdes. Ses bottes en cuir, noires elles aussi, se rendent à ses genoux, sont épaisses, lourdes, sur une plateforme solide et signalent leur origine, Doc Martens. Ses jeans sont serrés, comme une seconde peau, accentuant davantage sa maigreur et sa longueur. Il porte au cou un collier de chien où un crâne en acier flotte. Il a un anneau au nez. À chaque oreille, pend une effigie d'insecte mort. Il est frais rasé à la mâchoire et au crâne, avec une peau si pâle qui accentue le bleu-gris de ses yeux et toute cicatrice. Sa coupe de cheveux Mohawk avec ses pointes rousses bien dressées couronne le tout. Les cicatrices aux deux avant-bras révèlent une peau doublement épaissie à cause de multiples sutures. On peut déceler la place du tatouage au bras gauche.

Il me fait penser à un guerrier surhumain, tel Mad Max dans le film The Road Warrior, prêt à toute éventualité, mais surtout invulnérable. Est-ce cette seconde peau décrite par Anzieu, cette écorce dure qui l'aide à se défendre de la fragmentation interne? une affirmation agressive de sa virilité ? une quête réussie de démarcation individuelle, ou :

«[la] mise en évidence d'une esthétique et d'une morale de la présence, prothèse du moi éternellement en quête d'une 
incarnation provisoire pour assumer une trace significative de soi; il n'est plus question de se contenter du corps que l'on a mais d'en modifier les assises pour le compléter ou le rendre conforme à l'idée que l'on s'en fait. (Le Breton, 2004, 68)

Quelques passages de la Métamorphose de Kafka me viennent aussi en tête. Soudainement, un beau matin, Gregor se réveille de ses cauchemars diffus, pour se trouver transformé en un insecte monstrueux et grotesque qui terrifie et horrifie, se sentant lui-même emprisonné dans sa carapace, incapable de bouger et de laisser entrer en lui la nourriture et ainsi de se maintenir en vie. À prime abord, Kronos pourra-t-il prendre le risque de me laisser savoir tant bien que mal ce qui se passe en dedans de lui ou me gardera-t-il dehors, à distance, malgré son désir de contact, neutralisant ainsi mon savoir-pouvoir d'apprenti-sorcier?

Kronos n'a que de vagues souvenirs de sa mère, disparue lorsqu'il avait sept ou huit ans. Il ne sait pas si elle a quitté le domicile familial pour échapper à son mari, un homme qui veut tout contrôler ou si c'est lui qui l'a laissée, elle, victime d'une dépression sévère mal anesthésiée par l'usage régulier d'alcool et de drogues dures.

Son père ne consomme pas de drogues. Il se méfie de tout produit chimique qui pourrait influencer sa raison et son jugement. Il se consacre de plus en plus au survivalism - forme d'autarcie - pour pouvoir survivre à ce qu'il anticipe être l'éventuelle destruction partielle de la planète par une troisième guerre mondiale amorcée par les Russes. Ce père si méfiant de tout échange humain poussera Kronos et son petit frère à de multiples déménagements pour finalement se fixer en forêt profonde dans un isolement quasi-total où il se construit un abri antinucléaire. Malgré sa pratique extrême d'autarcie, le père succombe périodiquement à des régressions sévères où il perd tout contrôle, où il est en proie tour à tour au désespoir le plus sombre et à la rage la plus déchainée. Durant un de ces épisodes, il est admis à l'aile psychiatrique d'un hôpital général après avoir ingéré des clous et des morceaux de verre.

À l'adolescence, écoeuré d'être forcé à un isolement qu'il ne veut pas, Kronos prend la fuite seul, sans moyens, peut-être comme sa mère ou pour la rejoindre, et il retourne à la grande ville où il se sent chez lui. Cette information m'est transmise par bribes, durant les deux premières années, comme autant de morceaux mélangés d'un puzzle jamais fait, quelquefois sans affect d'une voix monotone et désincarnée, quelquefois dans ses dessins, quelquefois suivant un combat serré avec lui-même où le sang coule. Les éprouvés débordent brusquement et j'assiste à sa rage, son impuissance, son désespoir, ses terreurs, sa confusion et ses projections. Il a peur que je l'abandonne. Si je suis trop silencieuse, il a l'impression que je l'ai quitté, l'abandonnant à faire face seul à ce magma intérieur, informe et intemporel. Il devient un petit garçon terrifié quasi mutique. Si je parle trop, essayant trop fort de faire des liens, de mettre de l'ordre, il s'enrage et m'accuse de le contrôler, de lui imposer ma façon de penser les choses, comme son père. 
Puis il manque quelques séances avant de revenir. J'ai pensé par après, qu'il me protégeait, n'étant pas du tout sûr que je pourrais survivre à ses attaques et qu'il se punissait en se coupant pour m'avoir agressée.

Pendant un bon bout de temps, je me suis sentie dans un équilibre précaire où tout petit mouvement devait être pensé et planifié, comme si tous les deux, nous étions sur une corde raide où la moindre spontanéité pourrait nous faire basculer dans le vide.

En état d'hypervigilance en séance, je ressentais une fatigue intense par la suite. Avec de la pratique et du temps, comme l'athlète sur sa poutre de gymnastique, j'ai appris à lire son langage non verbal, à contenir plus ou moins son défoulement volcanique en le prévoyant dans mes propos avec lui, à lier et à transformer d'une certaine façon acceptable, significative pour lui, ce chaos interne pour qu'il puisse se trouver plus calme. Ses symptômes physiques s'améliorent: il mange mieux et plus souvent, il dort mieux et plus longtemps, il a moins de migraines, d'éruptions cutanées qui le démangent et de fièvres inexpliquées. Du moins pour un temps.

Alors que je souffle un peu et m'apprête à un temps de thérapie moins houleux, Kronos est hospitalisé pour une grippe sévère qui annonce la pneumonie. En convalescence, il est transféré à un hôpital psychiatrique à cause de ses périodes de dissociation qui inquiètent les médecins. Je le perds de vue pour plusieurs mois, parce que je ne travaille pas dans ce milieu hospitalier et une autre équipe de soins est mise en place.

Du côté professionnel, j'envisage une nouvelle occupation, puisque je suis reçue pédopsychiatre et du côté personnel, je vis les sévices d'une maladie sévère et chronique d'un parent. Ces changements provoquent une distance dans la relation thérapeutique lors des retrouvailles que ni lui, ni moi n'avons suffisamment abordée verbalement par après. Insuffisamment perlaborée, la séparation faiblit la relation et une phase beaucoup plus intellectuelle, plus cognitive s'instaure.

Vers la fin de la troisième année, Kronos me présente son amie. Elle semble plus jeune que lui. À la mode 'Goth'elle est vêtue exclusivement de noir, y compris le maquillage et le vernis à ongle. Ses cheveux mi-longs sont teints d'un blond presque blanc. Sa présence et la description que Kronos fait d'elle me font penser à une expression anglaise difficile à traduire - death warmed over. Étreindre la mort intimement...

M'ayant parlé de ses choix de carrière, il avait pensé devenir ambulancier, pour finalement entreprendre des études sur l'embaumement. Il porte maintenant un habit assez conventionnel et il a retiré tout objet qui avait choqué auparavant: son anneau au nez, ses chaînes, les insectes en effigie, le crâne en acier, la coupe Mohawk. Il lit Kübler-Ross et me parle de ses réflexions sur la perte et le deuil, et de l'aide à apporter aux personnes endeuillées. Il me parle aussi du corps mort, de son désir de rendre le cadavre à sa beauté d'avant la mort, pour que les gens puissent se souvenir de la personne telle qu'elle était en apparence lorsqu'elle était vivante. Faire à bas bruit le deuil de ses pertes d'objets? 
Il réussit bien ses études collégiales et fait son apprentissage de deux ans auprès d'un directeur de pompes funèbres en ville. Durant ce temps, on assiste à très peu d'urgences médicales ou psychiatriques.

Puis sans avertissement et sans préambule, il cesse de venir.

Il me laisse seule en compagnie de mes conjectures.

Inspirée par un article de Elmendorf (2007), j'en suis venue aussi à penser que les épisodes d'automutilation de Kronos servaient à signaler et à représenter de façon bien concrète sa désorganisation interne, son manque de cohésion, l'échec de sa peau comme surface-limite à contenir ce qui est dedans de ce qui est dehors. De voir s'écouler le sang et de ressentir une douleur physique le faisait revenir à la réalité. Ces épisodes servaient aussi peut-être à lui faire rechercher une ressource extérieure contenante dans la mesure où le milieu médical suturait ses plaies et détournait pour un temps sa haine contre lui-même. Se couper, c'était aussi ouvrir un peu cette deuxième peau psychique, devenue pratiquement une carapace impénétrable qui risquait d'entraîner l'épuisement, la famine, en ne laissant pas entrer les bons éléments nourrissant provenant de la relation.

De plus, la contrainte à répéter le poussait peut-être à revenir à une instance traumatique, je pense à celle d'être témoin impuissant de la perte de contrôle totale du père; et par identification à celui-ci de répéter ses mutilations à sa façon. Enfin ces épisodes servaient peut-être à se couper et tuer le père en lui, ressenti comme un objet persécuteur, envahissant et destructeur.

Je me demande en fait, qu'en est-il de ses identifications?

Dans un article sur le corps et sur le monde, Janine Chasseguet-Smirgel (2006) note que l'introjection est un processus supposé enrichir le sujet et est possible seulement si la relation avec l'objet a été suffisamment bonne et constante. Il s'ensuit que l'introjection du bon objet amène une vraie identification, supposée enrichir le moi Les investissements libidinaux et agressifs envers le père, dans ce cas, (et/ou de ses attributs, en particulier le pénis) se transforment en investissements narcissiques avec la même force et en côtoyant les investissements du moi.

Selon elle, un père insignifiant, méprisé par la mère - ou au contraire, un père brutal et rude, peut rendre le processus d'idéalisation difficile et de là entraver l'introjection du père et de ses attributs et ainsi perturber gravement le processus d'identification. Chasseguet-Smirgel prend pour modèle, en particulier, les écrits et la vie de Yukio Mishima pour formuler une hypothèse analytique de ce qui ce serait passé pour aboutir à son suicide spectaculaire sur la place publique en commettant le rituel japonais du seppuku.

Kronos aurait-il introjecté un père tout-puissant ou faute d'un bon modèle constant, se serait-il tourné vers lui-même et se serait-il mis en quête d'un corps invulnérable qu'il peut façonner et démarquer par le tatouage et l'apport vestimentaire et qu'il peut mettre à l'épreuve en se coupant ou en prenant des risques extrêmes?

Et qu'en est-il de sa relation avec sa mère? Ses premières expériences de vie ont-elles été suffisamment bonnes, avant qu'il ne la ressente comme non réceptive 
à lui et puis finalement absente? Et qu'en est-il du paradis perdu, celui de retourner dans la cavité première et de fusionner avec sa mère ? Est-ce la fusion avec une mère morte à toutes fins pratiques ? Doit-on aussi considérer ses gestes d'automutilation graves comme des gestes suicidaires pour rejoindre psychiquement sa mère et aussi paradoxalement avoir accès à l'immortalité, l'intemporalité des temps premiers? ou est-ce se tourner de plus en plus vers un nihilisme, cultiver le chaos, l'anarchie, le non-sens... se donner entièrement aux pensées 'punk'des années quatre-vingt?

Est-on jamais assez adepte pour s'établir comme analyste dans la coupure?

$$
\begin{array}{r}
\text { rose dymetryszyn } \\
77 \text { st-clair avenue, suite } 1807 \\
\text { toronto (ontario) } \\
\text { m4t 1m5 } \\
\text { dr.roseto@gmail.com }
\end{array}
$$

\section{Références}

Aulagnier, P., 1986 , Comment ne pas être persan? in Aulagnier, P., Un interprète en quête de sens. Paris, Petite Bibliothèque Payot, $2001,39-61$.

Chasseguet-Smirgel, J., 2006, The body and the world, in Zacharapoulos, E., éd. Beyond the Mind-Body Dualism : Psychoanalysis and the Human Body, Proceedings of the 6th Delphi International Psychoanalytic Symposium, 2004,International Congress Series, no. 1286, Amsterdam, Elsevier, 13-27.

Elmendorf, D., 2007, Containment And The Use of the Skin in Muller, J., Tillman, J., éds. In The Embodied Subject, Minding The Body In Psychoanalysis, New York, Jason Aronson, 81-91.

Gantheret, F., 1971, Remarques sur la place et le statut du corps en psychanalyse, in Lieux Du Corps, Nouvelle Revue de psychanalyse, $\mathrm{n}^{\circ}$ 3, Paris, Gallimard, 137-146.

Lebel, A., 2006, Angoisses Primitives : corps, pensée et identité, Colloque Annuel de la Société Psychanalytique de Montréal, avril 2006.

Le Breton, D., 2004-2005, Faire Euvre De Son Corps In Le Corps En Mouvement, Vie Des Arts, nº.197, 68-70. 